\title{
FRACTURE AND HEALING OF ELASTOMERS: A PHASE- TRANSITION THEORY AND NUMERICAL IMPLEMENTATION
}

\author{
Oscar Lopez-Pamies \\ Department of Civil and Environmental Engineering, University of Illinois at Urbana-Champaign, \\ MC-250 205 North Mathews Ave. Urbana, IL, civil@illinois.edu, http://cee.illinois.edu/
}

\begin{abstract}
Recent experiments, analogous to the classical experiments by Gent and collaborators but carried out at higher spatiotemporal resolution (of 1 micron in space and $60 \mathrm{~ms}$ in time), have provided a complete qualitative picture of the nucleation and the ensuing growth and interaction of internal cavities/cracks in elastomers subjected to externally applied quasi-static mechanical loads. In this talk, I will begin by presenting a continuum field theory seemingly capable to explain, describe, and predict all of the classical and recent experimental observations: from the nucleation of cavities/cracks, to their growth to micro-cracks, to their continued growth to macro-cracks, to the remarkable healing of some of the cracks. The theory rests on two central ideas. The first one is to view elastomers as solids capable to undergo finite deformations and capable also to phase transition to another solid of vanishingly small stiffness, whereas the forward phase transition serves to characterize the nucleation and propagation of fracture, the reverse phase transition characterizes the healing. The second central idea is to take the phase transition to be driven by the competition between a combination of strain energy and stress concentration in the bulk and surface energy on the created/healed new surfaces in the elastomer. In the second part of the talk, I will present a numerical implementation of the theory capable of efficiently dealing with large deformations, the typical near incompressibility of elastomers, and the large changes in the deformation field that can ensue locally in space and time from the nucleation of fracture. I will close by confronting its predictions with a number of recent experiments.
\end{abstract}

